\title{
The influence of patent foramen ovale on oxygen desaturation in obstructive sleep apnoea
}

\author{
M.C. Johansson*, P. Eriksson*, Y. Peker*, J. Hedner\#, L. Råstam and U. Lindblad" ${ }^{\top,+}$
}

ABSTRACT: Obstructive sleep apnoea (OSA) is associated with oxygen desaturation to a varying degree. A patent foramen ovale (PFO) may allow interatrial right-to-left shunting. The hypothesis of the current study was that oxygen desaturation will occur more often, in proportion to the frequency of respiratory disturbances, in OSA subjects with PFO than in those without.

In a group of 209 subjects diagnosed with OSA, the proportion of desaturation to respiratory events was calculated as the ratio of oxygen desaturation index (ODI)/apnoea-hypopnoea index $(\mathrm{AHI})$. A total of 15 cases with high proportional desaturation (ODI/AHI $\geqslant 0.66$ ) were individually matched with 15 controls with low proportional desaturation (ODI/AHI $\leqslant 0.33$ ), all without pulmonary disease. PFO was assessed with contrast transoesophageal echocardiography and considered large when $\geqslant 20$ bubbles passed over from the right to the left atrium after a single injection.

The prevalence of large PFO was nine out of $15(60 \%)$ in the high proportional desaturation group versus two out of $15(13 \%)$ in the low proportional desaturation group. The median number of passing bubbles was positively correlated to minimum oxygen saturation among those with PFO.

In conclusion, oxygen desaturation occurs more often, in proportion to the frequency of respiratory disturbances, in obstructive sleep apnoea subjects with a patent foramen ovale than in those without.

KEYWORDS: Echocardiography, heart septal defects, hypoxia, obstructive sleep apnoea, patent foramen ovale

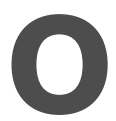
bstructive sleep apnoea (OSA) is a common disorder that affects $5-15 \%$ of the middle-aged population and has been identified as a risk factor for cardiovascular disease [1-3]. Patent foramen ovale (PFO) is also a common condition, present in $25 \%$ of the adult population, which constitutes a risk factor for cryptogenic stroke as well as a potential contributor to hypoxaemia in patients, both with and without pulmonary disease [4-7]. PFO and OSA are often considered to be two separate entities; however, as both have a high prevalence they sometimes co-exist and may influence the pathophysiology of each other. Obstructive apnoea provokes excessive intrathoracic pressure swings that greatly influence central haemodynamics, creating a right-to-left shunt through the PFO, which may cause significant desaturation [8-13]. This may explain why more severe desaturation than predicted from alveolar hypoventilation has been demonstrated in OSA patients [14-16]. The present study hypothesised that desaturation will occur more often proportionally to the frequency of respiratory disturbances in OSA subjects with PFO than in those without.

\section{METHODS}

\section{Selection of study population}

The study population was selected from a community-based sample described in the Skaraborg sleep study [17]. Briefly, 161 patients with and 183 subjects without hypertension were subjected to polysomnography, without consideration of any clinical symptoms of sleep apnoea. In total, 209 subjects were diagnosed with OSA. The oxygen desaturation index (ODI)/apnoeahypopnoea index (AHI) ratio was calculated for each of the 209 subjects. They were then ranked in accordance with their ODI/AHI ratios, divided into three groups and those subjects with the lowest and highest ratio $(\leqslant 0.33$ and $\geqslant 0.66$ ) were considered for inclusion. The 54 subjects with a ratio $\leqslant 0.33$ were defined as low proportional desaturation (PD) and those 57

\section{AFFILIATIONS}

*Sahlgrenska University Hospital/ Östra, and

\#Sahlgrenska University Hospital/ Sahlgrenska, Gothenburg, and

"Dept of Community Medicine, Lund University, Malmö, and +Skaraborg Institute, Skövde, Sweden.

\section{CORRESPONDENCE}

M.C. Johansson

Dept of Clinical Physiology

Sahlgrenska University Hospital/Östra

SE-416 85 Gothenburg

Sweden

Fax: 4631846334

E-mail: Magnus.C.Johansson@

vgregion.se

Received:

March 132006

Accepted after revision:

September 072006

SUPPORT STATEMENT

The study was supported by grants

from the Swedish Heart Lung

Foundation, the Västra Götaland

Region, and the Skaraborg Institute, Skövde, Sweeden. The sponsors of the study had no role in the study design, data collection, data analysis, data interpretation, or writing of the report. The corresponding author had full access to all the data in the study and had the final responsibility for the decision to submit for publication.

European Respiratory Journal Print ISSN 0903-1936 Online ISSN 1399-3003 
subjects with a ratio $\geqslant 0.66$, as high PD. Study participants were divided into pairs by contrasting their ratio, and 15 pairs with the highest and the lowest ratio were chosen with the aim of maximising the difference in desaturation between the subjects within each pair in order to test the study hypothesis. The subjects were matched for the presence of hypertension (with or without diabetes), body mass index (within $3 \mathrm{~kg} \cdot \mathrm{m}^{-2}$ ) and age (within $5 \mathrm{yrs}$ ). When more than one match was available, the minimum oxygen saturation and the mean overnight oxygen saturation were also considered in a manner that generated maximum difference. When a pair was split due to a participant being excluded or not giving consent, a second best match for the first subject was chosen. Following this procedure, 64 subjects were evaluated for participation before the final 15 matched pairs were finally identified. Exclusions were based on: death $(n=2)$, obstructive pulmonary disease $(n=7)$, other diseases $(n=3)$, or subjects not giving consent $(n=22)$. Characteristics of the subjects are described in table 1. All participants gave written informed consent to participate. The study was approved by the Gothenburg University human research ethics committee.

\section{Polysomnography}

The in-home, full-night polysomnography recording used a computerised recording system (Embla A10@); Embla, Reykjavik, Iceland), which consisted of the following: 1) sleep monitoring through three-channel electro-encephalography, two-channel electro-oculography, and one-channel submental electromyography; 2) bilateral tibial electromyography and a body-position detector; 3) two-lead ECG; and 4) respiration monitoring through an oro-nasal thermistor as well as nasal pressure sensor for apnoea-hypopnoea detection. Piezo crystal effort belts were used for thoracic-abdominal movement detection and a pulse oximeter (Embla Oximeter-XN; Embla) was applied. The sensors were applied and the equipment calibrated at the primary care centre by a certified sleep technician or specially trained local staff. Data were subsequently scored, based on 30-s epochs according to the

\begin{tabular}{|c|c|c|c|}
\hline & High PD\# & Low PD & p-value ${ }^{+}$ \\
\hline Subjects $n$ & 15 & 15 & \\
\hline Age yrs & $60.3 \pm 5.2$ & $61.0 \pm 5.8$ & NS \\
\hline Male & $12(80.0)$ & 8 (53.3) & 0.13 \\
\hline BMI $\mathbf{k g} \cdot \mathrm{m}^{-2}$ & $29.6 \pm 3.8$ & $29.8 \pm 3.7$ & NS \\
\hline Hypertension & 8 (53.3) & $8(53.3)$ & NS \\
\hline SBP $\mathrm{mmHg}$ & $141 \pm 16$ & $141 \pm 18$ & NS \\
\hline DBP $\mathrm{mmHg}$ & $81 \pm 10$ & $80 \pm 8$ & NS \\
\hline Diabetes & $2(13.3)$ & $2(13.3)$ & NS \\
\hline Smoking & $1(6.7)$ & $2(13.3)$ & NS \\
\hline ESS & $6.3 \pm 3.5$ & $6.7 \pm 3.4$ & NS \\
\hline
\end{tabular}

Data are presented as $\mathrm{n}, \mathrm{n}(\%)$ or mean $\pm \mathrm{SD}$, unless otherwise stated. PD: proportional desaturation; BMI: body mass index; SBP: systolic blood pressure; DBP: diastolic blood pressure; ESS: Epworth Sleepiness scale; NS: nonsignificant. *: oxygen desaturation index (ODI)/apnoea-hypopnoea index (AHI) $\geqslant 0.66 ; "$ : ODI/AHI $\leqslant 0.33$. All p-values are given unless $p>0.2$.
Rechtschaffen and Kales criteria [18]. An overall sleep stage report and accurate measures of respiratory events during the sleeping period were generated. Respiratory events were scored in accordance with guidelines for measurements in clinical research [19]. Obstructive apnoea (hypopnoea) was defined as a flat ( $\geqslant 40 \%$ reduction of $)$ nasal pressure signal accompanied by respiratory effort movements for $\geqslant 10 \mathrm{~s}$ and desaturation $\geqslant 3 \%$ from the immediately preceding baseline, or arousal. The definition of both apnoea and hypopnoea included the same requirement of $\geqslant 3 \%$ desaturation and/or arousal. The AHI was calculated to define the number of episodes of apnoea and hypopnoea per hour of sleep. OSA was defined as AHI $\geqslant 10$ obtained through sleep recording with a total sleep time of $\geqslant 4 \mathrm{~h}$. The ODI was defined as the number of episodes per hour of sleep with a reduction in saturation of $\geqslant 4 \%$ from baseline, and $\geqslant 10 \mathrm{~s}$.

Daytime sleepiness was assessed with the Epworth Sleepiness Scale, an eight-item self-administered questionnaire used for rating the likelihood of dozing in eight daily situations on a scale of $0-3$. The final score ranged from 0 (no daytime sleepiness) to 24 (maximum daytime sleepiness) [20].

\section{Spirometry}

Standard dynamic spirometry (Spirotrac; Vitalograph, Ennis, Ireland) was performed on the same day as the transoesophageal echocardiography (TE) examination in all subjects. Values were calculated as percentages of predicted values [21, 22]. Daytime percutaneous oxygen saturation was measured with the Ohmeda Biox 3740 (Ohmeda, Louisville, CO, USA).

\section{Transoesophageal contrast echocardiography}

From March to December 2003, one person (M. Johansson) performed all examinations. Subjects were instructed and trained to perform the Valsalva manoeuvre with $\geqslant 40 \mathrm{mmHg}$ for $8 \mathrm{~s}$. The achieved pressure was shown to the subject using a manometer. Multiplane TE was performed (Acuson Sequoia256 (Siemens, Munich, Germany) or General Electric Vivid 7 (General Electric, Fairfield, CT, USA) after mild sedation with midazolam and local pharyngeal anaesthesia (lidocaine). Colour Doppler with reduced pulse repetition frequency to $\sim 40 \mathrm{~cm} \cdot \mathrm{s}^{-1}$ and repeated contrast injections were used to detect PFO. A gelatin-based plasma expander $(3.5 \%$ polygelin; Aventis Pharma, Frankfurt am Main, Germany) and a small amount of air (5-10\% mixture) was agitated between two syringes mounted on a three-way stop-cock immediately before a bolus injection via a 20-gauge venous cannula [23]. All 2-mL injections were administered antecubitally while $10-\mathrm{mL}$ injections were administered via the foot vein, followed by a bolus injection of $5-10 \mathrm{~mL}$ saline. Contrast injections were given according to a standardised protocol that included injections during relaxed breathing in the supine and left lateral cubitus position and multiple provocations such as the Valsalva manoeuvre, coughing and bed tilt $[24,25]$. In order to reduce preload, nitroglycerin $(0.8 \mathrm{mg})$ was sprayed lingually during $10^{\circ}$ foot-down bed tilt, and contrast was injected antecubitally during relaxed breathing and Valsalva. Antecubital injections were made a few seconds after start of the Valsalva manoeuvre with the aim of maintaining strain for $\sim 10 \mathrm{~s}$ and making the septum primum bulge over towards the left atrium, at the same moment as the region in the right 
atrium adjacent to the fossa ovalis was filling with contrast [26]. When this failed, the timing of Valsalva versus contrast injection was adjusted and the procedure repeated. Foot vein injections were made a few seconds before the start of the Valsalva manoeuvre.

\section{PFO analysis}

The TE evaluation was performed off-line from Super-VHS video and blinded to the polysomnography results and patient group allocation. A PFO was defined as a minimum of three bubbles in the left atrium adjacent to the septum within three heartbeats from contrast filling of the right atrium [25]. The number of bubbles passing into the left atrium was estimated. A large PFO was defined as a minimum of 20 accumulated bubbles passing over after a single injection [5]. PFO analysis was made independently by two persons (M. Johansson and P. Eriksson) during 2003 and 2004. Disparities were settled by consensus with a third observer.

\section{Transthoracic echocardiography}

Standard echo-Doppler examinations were performed in all subjects. Left ventricular mass was calculated according to the corrected formula of the American Society of Echocardiography and indexed for body surface area. The longitudinal, myocardial, peak systolic and early diastolic velocities were assessed in the base of the left ventricular lateral wall and in the base of the right ventricular wall with spectral, pulsedwave tissue Doppler. The left ventricular ejection fraction was visually estimated. The systolic maximum tricuspid regurgitation gradient was assessed with and/or without signal amplification with agitated polygelin as echo contrast. Right atrial pressure was quantified on the basis of the respiratory variations of the inferior vena cava width and the right ventricular systolic pressure was calculated as the sum of the right atrial pressure and tricuspid regurgitation gradient. The left and right atrial area was measured in apical four-chamber view in end systole.

\section{Statistical analysis}

A minimum of $60 \%$ PFO prevalence in high PD subjects and a maximum of $15 \%$ in low PD subjects was hypothesised, and it was calculated that a sample of 15 pairs would give $80 \%$ power to detect any difference with a level of significance of $\mathrm{p}<0.05$. McNemar's two-tailed test was used for paired proportions. For comparison of the prevalence between groups, Fischer's two-tailed exact test was used; for quantitative parameters, an unpaired t-test was used and for correlation Pearson's test was used. A p-value $<0.05$ was considered statistically significant. All values are given as means $\pm \mathrm{SD}$, unless otherwise stated.

\section{RESULTS}

All 30 subjects completed contrast TE with 12-20 injections each. A PFO was found in $14(47 \%)$ subjects and was classified as small in three and large in 11 subjects. The PFO subjects received $17.6 \pm 1.6$ and the non-PFO subjects received $18.4 \pm 2.2$ injections. A large PFO was found in nine out of the $15(60 \%)$ high PD cases but only in two out of the 15 (13\%) low PD controls $(p=0.02)$, as shown in figure 1 . Furthermore, the PFOs were found in individuals with a large range of AHI values as shown in figure 2. The paired distribution showed a higher prevalence of large PFOs in high PD cases than in low PD controls. There were eight pairs in which only the high PD case had a large PFO, one pair in which both had a PFO, five pairs in which no large PFO was found, while in one pair only the low PD control had a large PFO $(p<0.04)$. The paired distribution regarding all-size $\mathrm{PFO}$ did not reach a statistically significant difference $(p=0.07)$. The predictive value of the ODI/AHI ratio for PFO detection was calculated. A high ratio $(\geqslant 0.66)$ had a sensitivity of $82 \%$ and a positive predictive value of $60 \%$. A low ratio $(\leqslant 0.33)$ had a specificity of $68 \%$ and a negative predictive value of $87 \%$. The spirometry and polysomnography data for the groups are shown in table 2. As expected, the ODI is higher in high PD cases than in low PD controls, but the significant difference is not explained by AHI. In fact, the AHI was not significantly different between groups. No significant difference in AHI, apnoea index or apnoea duration was found regarding the presence or absence of a large PFO. However, the ODI/AHI ratio in large PFO subjects was twice that found in subjects without a large PFO. The ODI/AHI ratio, but not the minimum oxygen saturation and ODI per se, was fairly well correlated to a large PFO $(r=0.55$,

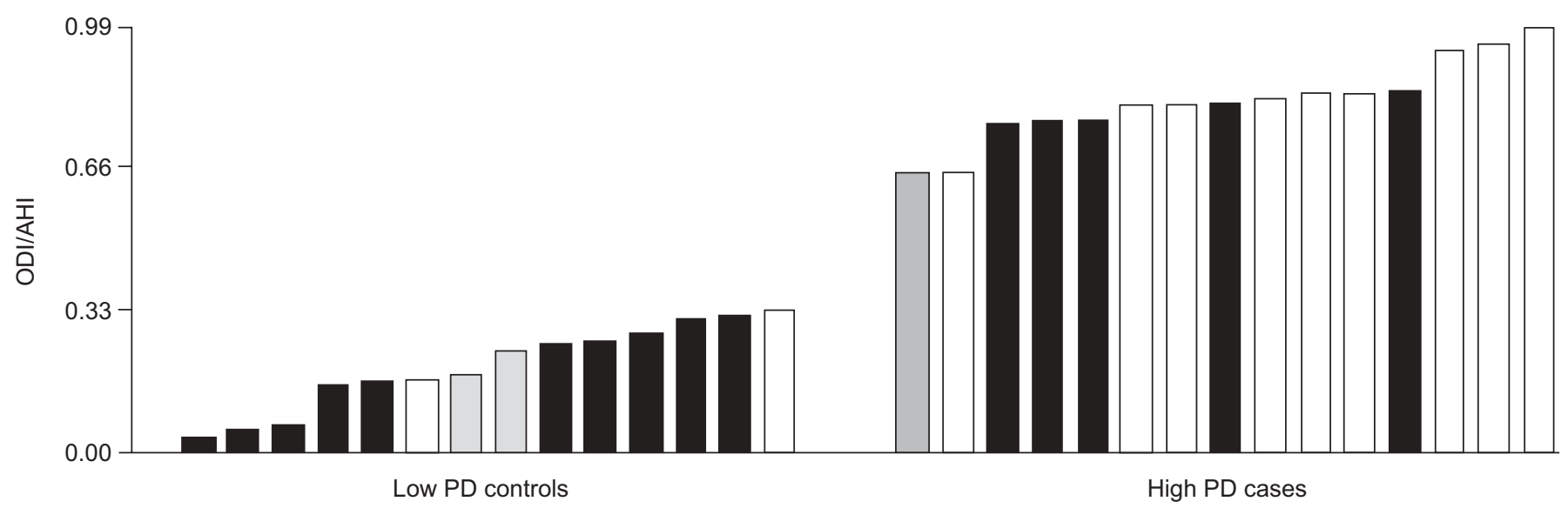

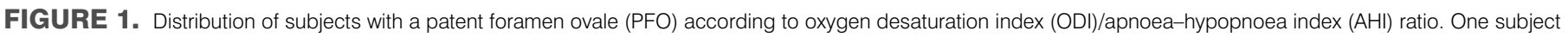

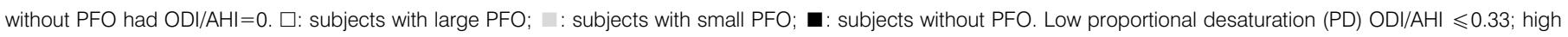
$\mathrm{PD}: \mathrm{ODI} / \mathrm{AHI} \geqslant 0.66$; 


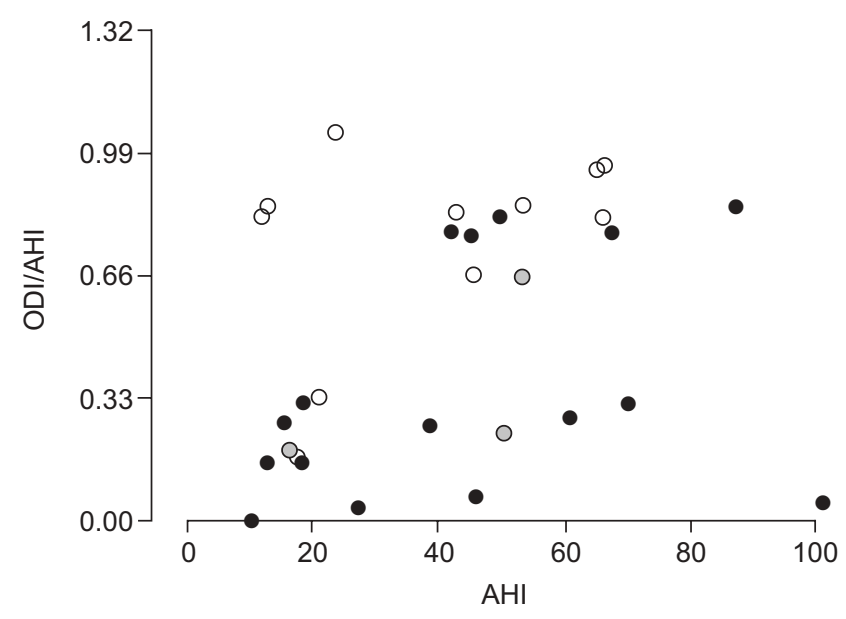

FIGURE 2. Correlation of oxygen desaturation index (ODI)/apnoea-hypopnoea index $(\mathrm{AHI})$ ratio and $\mathrm{AHI}$ among subjects with (large $(\bigcirc)$ or small $(\bigcirc)$ ) and without (-) a patent foramen ovale (PFO). It was more likely that a subject in the upper part of the graph would have a PFO than a subject in the lower part, irrespective of $\mathrm{AHI}$ value.

$p=0.02)$. The median number of bubbles passing into the left atrium after an injection was significantly correlated among all 30 subjects with nocturnal minimum oxygen saturation $(\mathrm{r}=0.36, \mathrm{p}=0.05)$ and among the 14 subjects with a PFO $(\mathrm{r}=0.62, \mathrm{p}=0.02)$. However, age, body mass index, hypertension, diabetes and blood pressure did not differ in accordance with the PFO diagnosis. The measured echo parameters did not differ between groups, as shown in table 3 . The characteristics of patients with high PD are shown in table 4; patients with a high PD without a PFO had significantly more hypopnoeas that those with PFO.

\section{DISCUSSION}

To the best of the current authors' knowledge, this is the first study demonstrating an association between nocturnal desaturations in OSA and the existence of PFO. In order to discriminate between desaturation caused by veno-arterial admixture and desaturation caused by apnoea-hypopnoearelated interruption in alveolar ventilation, the ODI/AHI ratio was calculated for each OSA subject. This ratio is a novel construction that consists of two well-defined parameters with the same denomination: number per hour of sleep. The ODI and AHI values were fairly well correlated with each other among those 209 OSA subjects from whom the 30 study subjects were selected $(\mathrm{r}=0.8, \mathrm{p}<0.01)$. Also in the literature, ODI is generally considered to be positively correlated with AHI in OSA subjects [27]. Due to this correlation, the ODI/AHI ratio was considered to be a factor that roughly corrects the desaturation frequency for the apnoea-hypopnoea frequency and that its variation reveals that factors other than ventilation could be involved. Although the correlation is good in a group of OSA subjects, large variations are actually found when AHI and ODI are compared between individual subjects [27]. In the whole group of 209 subjects with OSA, the AHI values were generally higher than the ODI values, with a mean difference of 15.8 and a considerable scatter (SD 13.7). According to the present hypothesis, a subject with an ODI of 16 and AHI of 20 (ODI/AHI 0.8) would be more likely to have a PFO than a subject with an ODI of 10 and an AHI of 40 (ODI/AHI 0.25),

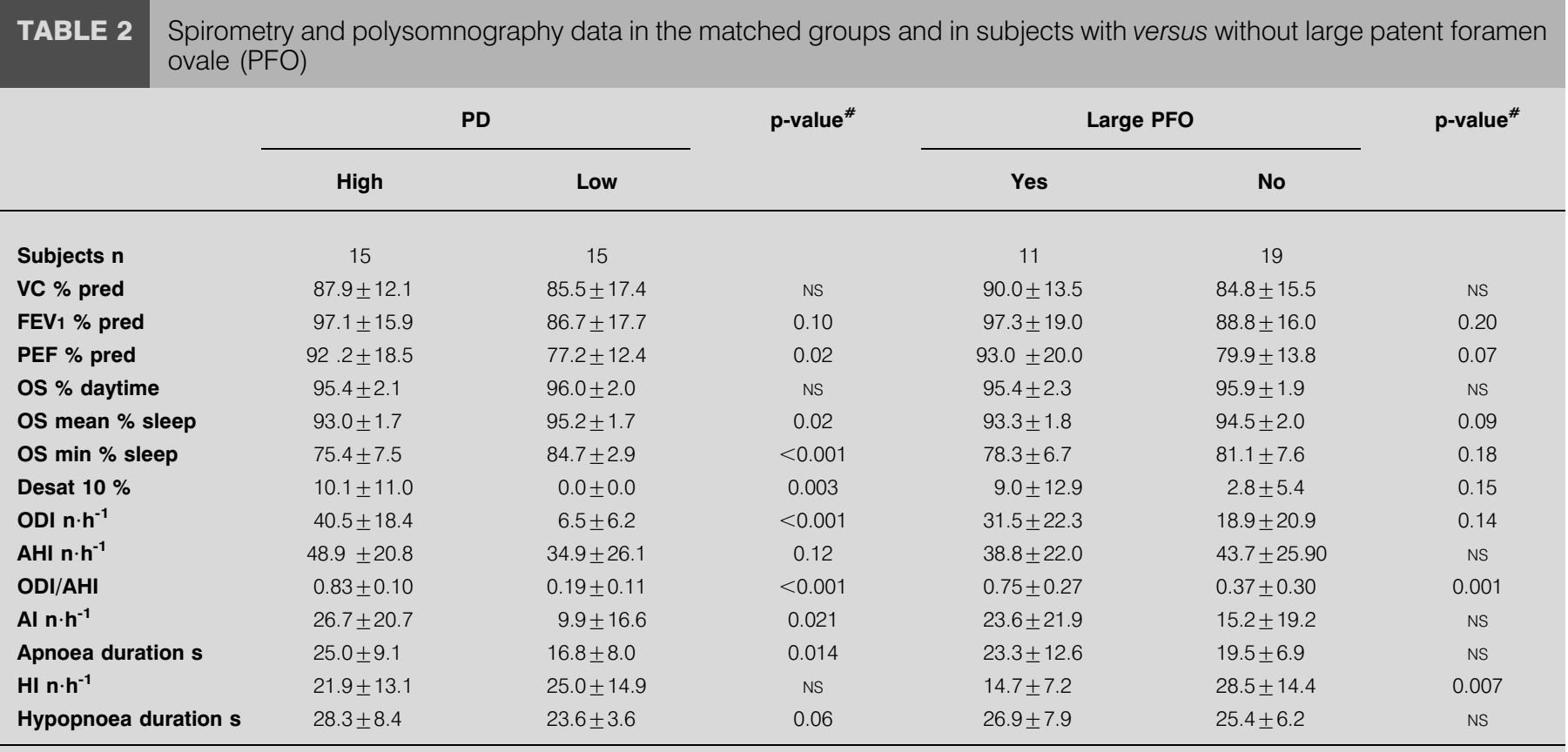

Data are presented as $n$ or mean \pm SD, unless otherwise stated. PD: proportional desaturation; VC: vital capacity; \% pred: \% predicted; FEV1: forced expiratory volume in one second; PEF: peak expiratory flow. OS: oxygen saturation percutaneously measured; Desat 10: episodes per hour of sleep with desaturation $>10 \%$; ODI: oxygen desaturation index; AHI: apnoea-hypopnoea index; Al: apnoea index (number of apnoeic episodes per hour of sleep); HI: hypopnoea index (number of episodes of hypopnoea per hour of sleep); Ns: nonsignificant. All p-values are given unless $p<0.2$. 


\begin{tabular}{|c|c|c|c|c|c|c|}
\hline \multirow[t]{3}{*}{ TABLE 3} & \multicolumn{6}{|c|}{$\begin{array}{l}\text { Echocardiography data in the matched groups } \\
\text { and in subjects with versus without large patent } \\
\text { foramen ovale (PFO) }\end{array}$} \\
\hline & \multicolumn{2}{|c|}{ PD } & \multirow[t]{2}{*}{ p-value ${ }^{\#}$} & \multicolumn{2}{|c|}{ Large PFO } & \multirow[t]{2}{*}{ p-value } \\
\hline & High & Low & & Yes & No & \\
\hline Subjects n & 15 & 15 & & 11 & 19 & \\
\hline LVMI $g \cdot m^{-2}$ & $123 \pm 26$ & $122 \pm 28$ & NS & $117 \pm 26$ & $126 \pm 28$ & NS \\
\hline LVEF \% & $58 \pm 3$ & $58 \pm 4$ & NS & $59 \pm 3$ & $59 \pm 4$ & NS \\
\hline $\mathrm{Sm} \mathrm{cm} \cdot \mathrm{s}^{-1}$ & $13.2 \pm 4.0$ & $12.9 \pm 2.6$ & NS & $12.8 \pm 2.9$ & $13.5 \pm 4.1$ & NS \\
\hline $\mathrm{Em} \mathrm{cm} \cdot \mathrm{s}^{-1}$ & $12.6 \pm 2.9$ & $12.3 \pm 2.2$ & NS & $12.3 \pm 2.9$ & $12.6 \pm 2.4$ & NS \\
\hline $\mathrm{E} / \mathrm{Em}$ & $5.7 \pm 1.3$ & $6.0 \pm 1.4$ & NS & $5.9 \pm 1.2$ & $5.8 \pm 1.4$ & NS \\
\hline Sm,RV & $18.6 \pm 6.0$ & $18.0 \pm 5.1$ & NS & $19.3 \pm 5.3$ & $17.8 \pm 4.8$ & NS \\
\hline Em,RV & $16.9 \pm 6.8$ & $16.7 \pm 3.8$ & NS & $16.6 \pm 8.0$ & $17.0 \pm 3.5$ & NS \\
\hline ERV/Em.RV & $3.3 \pm 1.1$ & $3.1 \pm 0.7$ & NS & $3.2 \pm 1.1$ & $3.2 \pm 0.8$ & NS \\
\hline RAP $\mathrm{mmHg}$ & $6.0 \pm 2.1$ & $5.0 \pm 0$ & 0.08 & $5.5 \pm 1.5$ & $5.5 \pm 1.6$ & NS \\
\hline RVSP $\mathrm{mmHg}$ & $27.8 \pm 6.5$ & $26.8 \pm 3.3$ & NS & $27.3 \pm 7.2$ & $27.3 \pm 3.6$ & NS \\
\hline LA area & $20.4 \pm 4.6$ & $21.5 \pm 4.3$ & NS & $19.4 \pm 4.0$ & $21.8 \pm 4.5$ & NS \\
\hline RA area & $16.9 \pm 3.6$ & $16.2 \pm 2.7$ & NS & $16.5 \pm 3.1$ & $16.5 \pm 3.2$ & NS \\
\hline \multicolumn{7}{|c|}{ 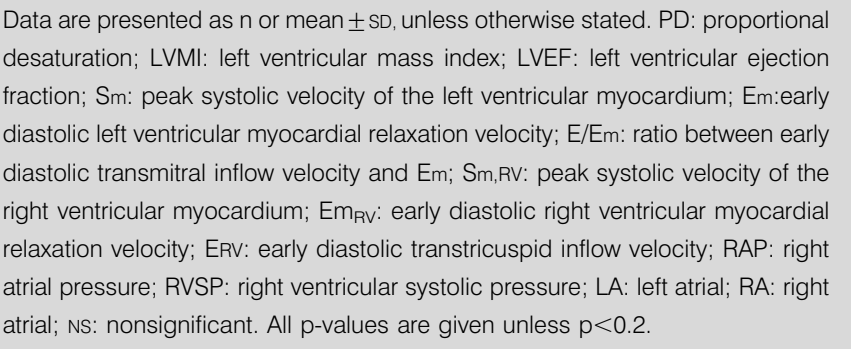 } \\
\hline
\end{tabular}

even though the OSA is more severe in this latter subject. As shown in table 2, pulmonary function was significantly better in high PD cases than in low PD controls, supporting the hypothesis that other factors besides respiratory factors are involved. Moreover, the variation in ODI/AHI ratio between groups was not explained by the AHI. However, the power of the ODI/AHI ratio to predict shunt-related desaturation is limited. The magnitude of desaturation from an interatrial shunt will depend on the size of the PFO and the interatrial pressure relation. The potential opening diameter of the PFO ranges $\geqslant 1-19 \mathrm{~mm}$ [4]. Since the number of bubbles passing through is only a rough estimate of the diameter during balloon sizing, and no catheterisation was performed, the exact maximum opening diameter is not known [28]. The interatrial pressure relationship will depend on the degree of right-heart loading during obstructive apnoea but also on concomitant left-heart condition [23]. Shunt diagnosis was only performed with the subjects awake, for which reason the actual degree of shunting during sleep is unknown. In order to overcome this weakness the frequency of moderate desaturation $(>4 \%)$ rather than maximum desaturation was focused upon. BEELKE et al. [29] found right-to-left shunting in nine out of 10 PFO subjects during obstructive apnoea lasting longer than $17 \mathrm{~s}$ but not during hypopnoea. That study exclusively included OSA subjects with apnoea-hypopnoea and concomitant $4 \%$ desaturation, which would correspond to the high PD cases in the present study. One of the two low PD controls with PFO in the present study had only hypopnoeic and no apnoeic

\begin{tabular}{lccc} 
TABLE 4 & $\begin{array}{l}\text { Characteristics of high proportional desaturation } \\
\text { (PD) cases according to presence of a large } \\
\text { patent foramen ovale (PFO) }\end{array}$ \\
Large PFO & Yes & No & p-value \\
\hline Subjects $\mathbf{n}$ & 9 & 6 & \\
ODI/AHI & $0.86 \pm 0.11$ & $0.78 \pm 0.07$ & 0.08 \\
AHI $\mathbf{n}$ & $43 \pm 22$ & $58 \pm 17.0$ & 0.18 \\
HI $\mathbf{n}$ & $14.0 \pm 7.7$ & $34 \pm 10.3$ & 0.003 \\
Al $\mathbf{n}$ & $28.6 \pm 21.4$ & $23.8 \pm 21.3$ & $\mathrm{NS}$ \\
Hypopnoea $\mathbf{~ m i n} \cdot \mathbf{h}^{-\mathbf{1}}$ & $5.8 \pm 2.4$ & $14.8 \pm 5.1$ & 0.006 \\
Apnoea $\mathbf{m i n} \cdot \mathbf{h}^{-\mathbf{1}}$ & $14.0 \pm 12.9$ & $9.2 \pm 8.9$ & $\mathrm{NS}$ \\
\hline
\end{tabular}

Data are presented as $\mathrm{n}$ or mean $\pm \mathrm{SD}$, unless otherwise stated. ODI: oxygen desaturation index; AHI: apnoea-hypopnoea index; HI: hypopnoea index (number of episodes of hypopnoea per hour of sleep); Al: apnoea index (number of apnoeic episodes per hour of sleep); Ns: nonsignificant. All p-values are given unless $p<0.2$.

events, while the other had only three episodes of apnoea per hour of sleep. The current results are in contrast, in part, to the study of SHANOUDY et al. [12], which showed a generally increased prevalence of PFO in OSA, but did not consider the degree of desaturation in relation to apnoeic events. The present study showed a low prevalence of only $13 \%$ in the low PD group. It also seems logical that PFO is not a cause of upper airway obstruction, but its valve-like function permits unidirectional right-to-left shunting during right-heart loading, such as that occurring during obstructive apnoea $[8,9,29]$. The analysis of PFO is not always distinct. There was disagreement in the analysis of one large PFO and three small PFOs; however, they were all solved through consensus. This is in concordance with CABANES et al. [25] who found considerable variation in small-PFO analysis with only a few bubbles passing to the left atrium. However, the clinical significance of these small shunts is probably very limited [5]. In the current study, the PFO channel was visualised in nine subjects, all with large PFO, whereas in the other subjects, the exact location of the passage could not be visualised. Another route of contrast passage could hypothetically be intrapulmonary shunts, but this is probably not the case as contrast appeared in the left atrium adjacent to the septum within three heartbeats from contrast filling of the right atrium. The current sampling and classification procedure may have been skewed towards high AHI values in this population, as the definition of respiratory events was based on nasal pressure cannula recording. Moreover, obstructive events were also scored when respiratory events included arousal but not necessarily desaturation. This practice may also have elevated the AHI value in patients with minor desaturation but frequent arousal responses. Apnoea duration and the relationship between episodes of apnoea and hypopnoea may also have introduced a confounding influence. In calculating ODI/AHI, apnoeas and hypopnoeas were counted together, although shunting seems only to occur during apnoeas [29]. The high PD cases without PFO had significantly more hypopnoeas than those with PFO (table 4). Frequent hypopnoeas in OSA subjects have been associated with reduced hypoxic ventilatory drive [30]. This may cause a high ODI/AHI ratio because of reduced ventilation between 
events and relatively low respiratory event scoring, as the baseline respiratory flow is also reduced.

The present study supports the hypothesis that interatrial shunting gives a substantial increase in the number of desaturations in OSA subjects with PFO. Moreover, this may be the mechanism that explains the increased risk of stroke that is seen in OSA [31]. If this link could be established, percutaneous closure of a PFO may be a potential treatment option in the future [32]. A strength of the present study is that it was based on a cross-sectional population sample randomly selected for polysomnography without prior knowledge of sleep disturbances. Subjects diagnosed with OSA were considered for inclusion on the basis of their ODI/AHI ratio and with obstructive pulmonary disease as the only exclusion criterion. Although the study group was small, the findings may therefore be regarded as reasonably applicable to a general population of OSA patients with healthy lungs.

In summary, oxygen desaturation occurs more often in proportion to the frequency of respiratory disturbances in obstructive sleep apnoea subjects with a patent foramen ovale than in those without. The oxygen desaturation index/apnoeahypopnoea index ratio might be a clinically useful screening tool, which is able to select obstructive sleep apnoea subjects with a high likelihood of a patent foramen ovale.

\section{ACKNOWLEDGEMENTS}

The authors would like to thank technicians S. Eriksson, A-M. Edvardsson and S. Melander (Clinical Physiology, SU/ Östra, Gothenburg, Sweden) for their excellent assistance with the echo exams, and to A. Mehner and A. Segerblom for excellent technical and secretarial assistance at the Health Care Centre in Skara (Sweden).

\section{REFERENCES}

1 Young T, Palta M, Dempsey J, Skatrud J, Weber S, Badr S. The occurrence of sleep-disordered breathing among middle-aged adults. N Engl J Med 1993; 328: 1230-1235.

2 Peker Y, Hedner J, Norum J, Kraiczi H, Carlson J. Increased incidence of cardiovascular disease in middle-aged men with obstructive sleep apnea: A 7-Year Follow-up. Am J Respir Crit Care Med 2002; 166: 159-165.

3 Dyugovskaya L, Lavie P, Hirsh M, Lavie L. Activated CD8+ T-lymphocytes in obstructive sleep apnoea. Eur Respir J 2005; 25: 820-828.

4 Hagen PT, Scholz DG, Edwards WD. Incidence and size of patent foramen ovale during the first 10 decades of life: an autopsy study of 965 normal hearts. Mayo Clin Proc 1984; 59: 17-20.

5 Webster MW, Chancellor AM, Smith HJ, et al. Patent foramen ovale in young stroke patients. Lancet 1988; 2: 11-12.

6 Hacievliyagil SS, Gunen H, Kosar FM, Sahin I, Kilic T. Prevalence and clinical significance of a patent foramen ovale in patients with chronic obstructive pulmonary disease. Respiratory Medicine 2006; 100: 903-910.

7 Godart F, Rey C, Prat A, et al. Atrial right-to-left shunting causing severe hypoxaemia despite normal right-sided pressures. Report of 11 consecutive cases corrected by percutaneous closure. Eur Heart J 2000; 21: 483-489.
8 Shiomi T, Guilleminault C, Stoohs R, Schnittger I. Leftward shift of the interventricular septum and pulsus paradoxus in obstructive sleep apnea syndrome. Chest 1991; 100: 894-902.

9 Brinker JA, Weiss JL, Lappe DL, et al. Leftward septal displacement during right ventricular loading in man. Circulation 1980; 61: 626-633.

10 Tilkian AG, Guilleminault C, Schroeder JS, Lehrman KL, Simmons FB, Dement WC. Hemodynamics in sleepinduced apnea. Studies during wakefulness and sleep. Ann Intern Med 1976; 85: 714-719.

11 Iwase N, Kikuchi Y, Hida W, et al. Effects of repetitive airway obstruction on $\mathrm{O} 2$ saturation and systemic and pulmonary arterial pressure in anesthetized dogs. Am Rev Respir Dis 1992; 146: 1402-1410.

12 Shanoudy H, Soliman A, Raggi P, Liu JW, Russell DC, Jarmukli NF. Prevalence of patent foramen ovale and its contribution to hypoxemia in patients with obstructive sleep apnea. Chest 1998; 113: 91-96.

13 Beelke M, Angeli S, Del Sette M, et al. Prevalence of patent foramen ovale in subjects with obstructive sleep apnea: a transcranial Doppler ultrasound study. Sleep Medicine 2003; 4: 219-223.

14 Bradley TD, Martinez D, Rutherford R, et al. Physiological determinants of nocturnal arterial oxygenation in patients with obstructive sleep apnea. J Appl Physiol 1985; 59: 1364-1368.

15 Appelberg J, Nordahl G, Janson C. Lung volume and its correlation to nocturnal apnoea and desaturation. Respir Med 2000; 94: 233-239.

16 Findley LJ, Ries AL, Tisi GM, Wagner PD. Hypoxemia during apnea in normal subjects: mechanisms and impact of lung volume. J Appl Physiol 1983; 55: 1777-1783.

17 Hedner J, Bengtsson-Bostrom K, Peker Y, Grote L, Rastam L, Lindblad U. Hypertension prevalence in obstructive sleep apnoea and sex: a population-based case-control study. Eur Respir J 2006; 27: 564-570.

18 Rechtschaffen A, Kales A. A manual for standardized terminology, techniques, and scoring system for sleep stages of human subjects. Washington DC, US Public Health Service, US Government Printing Office, 1968.

19 Sleep-related breathing disorders in adults: recommendations for syndrome definition and measurement techniques in clinical research. The Report of an American Academy of Sleep Medicine Task Force. Sleep 1999; 22: 667-689.

20 Johns MW. A new method for measuring daytime sleepiness: the Epworth sleepiness scale. Sleep 1991; 14: 540-545.

21 Berglund E, Birath G, Bjure J, et al. Spirometric studies in normal subjects. I. Forced expirograms in subjects between 7 and 70 years of age. Acta Med Scand 1963; 173: 185-192.

22 Vale JR. Maximum expiratory flow velocity. Nord Med 1963; 70: 848-851.

23 Siostrzonek $\mathrm{P}$, Lang $\mathrm{W}$, Zangeneh $\mathrm{M}$, et al. Significance of left-sided heart disease for the detection of patent foramen ovale by transesophageal contrast echocardiography. J Am Coll Cardiol 1992; 19: 1192-1196.

24 Kerr A. A new method for formaen ovale assessment by echo: bed-tilt and transmitral Doppler. J Am Soc Echocardiogr 1999; 12: 407A. 
25 Cabanes L, Coste J, Derumeaux G, et al. Interobserver and intraobserver variability in detection of patent foramen ovale and atrial septal aneurysm with transesophageal echocardiography. J Am Soc Echocardiogr 2002; 15: 441-446.

26 De Castro S, Cartoni D, Fiorelli M, et al. Morphological and functional characteristics of patent foramen ovale and their embolic implications. Stroke 2000; 31: 2407-2413.

27 Oeverland B, Skatvedt O, Kvaerner KJ, Akre H. Pulseoximetry: sufficient to diagnose severe sleep apnea. Sleep Med 2002; 3: 133-138.

28 Schuchlenz HW, Weihs W, Beitzke A, Stein JI, Gamillscheg A, Rehak P. Transesophageal echocardiography for quantifying size of patent foramen ovale in patients with cryptogenic cerebrovascular events. Stroke 2002; 33: 293-296.
29 Beelke M, Angeli S, Del Sette M, et al. Obstructive sleep apnea can be provocative for right-to-left shunting through a patent foramen ovale. Sleep 2002; 25: 856-862.

30 Kunitomo F, Kimura H, Tatsumi K, et al. Abnormal breathing during sleep and chemical control of breathing during wakefulness in patients with sleep apnea syndrome. Am Rev Respir Dis 1989; 139: 164-169.

31 McArdle N, Riha RL, Vennelle M, et al. Sleep-disordered breathing as a risk factor for cerebrovascular disease: a case-control study in patients with transient ischemic attacks. Stroke 2003; 34: 2916-2921.

32 Johansson M, Soderberg B, Eriksson P. Availability of percutaneous closure for an adult population with interatrial shunts. Cardiology 2003; 99: 85-89. 